MATHEMATICS OF COMPUTATION

Volume 74, Number 250, Pages 1001-1002

S 0025-5718(04)01673-4

Article electronically published on May 25, 2004

\title{
A PRIMITIVE TRINOMIAL OF DEGREE 6972593
}

\author{
RICHARD P. BRENT, SAMULI LARVALA, AND PAUL ZIMMERMANN
}

Abstract. The only primitive trinomials of degree 6972593 over GF(2) are $x^{6972593}+x^{3037958}+1$ and its reciprocal.

\section{INTRODUCTION}

This note extends the computation [2, to which we refer for motivations, definitions, historical comments and additional references. All polynomials are assumed to be in $Z_{2}[x]$. When considering trinomials $T(x)=x^{r}+x^{s}+1$, we assume that $1 \leq s \leq\lfloor r / 2\rfloor$, so we disregard the reciprocal trinomial $x^{r} T(1 / x)=x^{r}+x^{r-s}+1$.

We restrict our attention to Mersenne exponents $r$, for which $2^{r}-1$ is prime. Trinomials $x^{r}+x^{s}+1$ whose degree is a Mersenne exponent $r \leq 3021377$ are considered in [2, 3, 4]. Here we consider the next Mersenne exponent $r=6972593$. According to the GIMPS project [7], 6972593 is the only Mersenne exponent in the interval $\left(3021377,10^{7}\right)$.

\section{Computational Results}

Using the algorithm of [2, §4], a search for irreducible trinomials of degree $r=6972593$ was started in February 2001 and completed in July 2003. Sieving eliminated all but $236244(6.78 \%)$ of the $\lfloor r / 2\rfloor=3486296$ candidate trinomials and took about $5 \%$ of the total time. In most cases we sieved up to degree 26 .

For the 236244 candidate trinomials $T(x)$ not eliminated by sieving, we computed $x^{2^{r}} \bmod T(x)$ : since $r$ is prime, $T(x)$ is irreducible iff $x^{2^{r}}=x \bmod T(x)$. In some cases we tested the reciprocal of $T(x)$ instead of $T(x)$; see [2, Thm. 2 and $\S 4$ ].

One primitive trinomial,

$$
T(x)=x^{6972593}+x^{3037958}+1,
$$

was found on August 31, 2002. Our computations show that this is the only primitive trinomial of degree 6972593, apart from its reciprocal.

The computation was performed on an average of about 300 processors and took approximately 230000 Mips-years (17.8 times as long as for $r=3021377)$.

\section{Checking the Results}

It is important to check the results of such a long computation to detect human, software and/or hardware errors [4, 5, 7]. Most software that can be used

Received by the editor August 26, 2003 and, in revised form, October 6, 2003.

2000 Mathematics Subject Classification. Primary 11B83; Secondary 11-04, 11N35, 11R09, 11T06, 11Y55, 12-04.

Key words and phrases. Irreducible trinomials, primitive trinomials, Mersenne numbers. 
to compute irreducible/primitive trinomials is impractical for degrees as large as 6972593 because of inefficient use of memory or nonoptimized algorithms. NTL [6] is the only general-purpose package that we have found capable of checking the irreducibility of a trinomial of degree 6972593 over $Z_{2}$, and NTL takes three times longer than our program irred (13 hours versus 4.33 hours on an $833 \mathrm{Mhz}$ Alpha EV68 to verify our primitive trinomial).

The $\log$ files for $r=6972593$ and other Mersenne exponents less than $10^{7}$ are available on our website [1, along with details of the checks performed. Any discrepancies found during checking will be reported there.

\section{ACKNOWLEDGMENTS}

The following institutions and individuals contributed to the computing task (approximate percentages in parentheses): Centre Informatique National de l'Enseignement Supérieur (30\%); Oxford Supercomputing Centre (26\%); Oxford Centre for Computational Finance (19\%); Oxford University Computing Laboratory (14\%); Australian Partnership for Advanced Computing, courtesy of Brendan McKay (9\%); Juan Luis Varona (1.3\%); Barry Mead (0.4\%); Nicolas Daminelli $(0.2 \%)$; and Nate Begeman (0.1\%). Nate also ported our program to the Mac G4, using Motorola's AltiVec ISA extension to the PowerPC processor. We thank Philippe Falandry for assistance in compiling our program on the IBM SP in 64bit mode, Julian Seward and Andrew Tridgell for their assistance in running the valgrind simulator, Victor Shoup for his NTL package [6], George Woltman and the GIMPS project 7] for providing information on Mersenne exponents, and an anonymous referee for encouraging us to make the text more succinct. The first author acknowledges the support of EPSRC Grant GR/N35366/01.

\section{REFERENCES}

[1] R. P. Brent, Search for primitive trinomials, http://www.comlab.ox.ac.uk/oucl/work/ richard.brent/trinom.html.

[2] R. P. Brent, S. Larvala and P. Zimmermann, A fast algorithm for testing reducibility of trinomials mod 2 and some new primitive trinomials of degree 3021377, Math. Comp. 72 (2003), 1443-1452.

[3] R. P. Brent and P. Zimmermann, Algorithms for finding almost irreducible and almost primitive trinomials, in Primes and Misdemeanours: Lectures in Honour of the Sixtieth Birthday of Hugh Cowie Williams, Fields Institute, Toronto, 2004, to appear.

[4] T. Kumada, H. Leeb, Y. Kurita and M. Matsumoto, New primitive $t$-nomials $(t=3,5)$ over GF(2) whose degree is a Mersenne exponent, Math. Comp. 69 (2000), 811-814; Corrigenda: ibid 71 (2002), 1337-1338. MR 2000i:11183; MR 2003c:11153

[5] T. J. Nicely, Enumeration to $10^{14}$ of the twin primes and Brun's constant, Virginia Journal of Science 46 (1995), 195-204. MR 97e:11014

[6] V. Shoup, NTL: A library for doing number theory (version 5.3.1), http://www.shoup.net/ ntl/.

[7] G. Woltman et al., GIMPS, The Great Internet Mersenne Prime Search, http://www. mersenne.org/.

Oxford University COMPuting Laboratory, OXford OX1 3QD, United Kingdom

E-mail address: trinomials@rpbrent.co.uk

Helsinki University of Technology, Espoo, Finland

E-mail address: slarvala@cc.hut.fi

LORIA/INRIA Lorraine, 615 RUe du JARdin botanique, BP 101, F-54602 Villers-LèsNANCY, FRANCE

E-mail address: Paul.Zimmermann@loria.fr 